\title{
INTERPRETAÇÃO DO MECANISMO DE DIFUSÃO NO PROCESSO GÁS-SÓLIDO DA REAÇÃO DE DESSULFURIZAÇÃO
}

\section{Daniela Andresa Mortari*, Paula Manoel Crnkovic, Ivonete Ávila e Fernando Eduardo Milioli}

Departamento de Engenharia Mecânica, Escola de Engenharia de São Carlos, Universidade de São Paulo, CP 780, 13560-970 São Carlos - SP, Brasil

Recebido em 20/1/10; aceito em 16/6/10; publicado na web em 22/9/10

\begin{abstract}
INTERPRETATION OF THE MECHANISM DIFFUSION IN THE GAS-SOLID PROCESS OF THE DESSULFURIZATION REACTION. The present paper aims to interpret the $\mathrm{SO}_{2}$ diffusion mechanism process for two different limestones: a calcite and a dolomite. In previous study, the apparent activation energies for sulfation reaction were between 3.03 and $4.45 \mathrm{~kJ} \mathrm{~mol}^{-1}$ for the calcite, and $11.24 \mathrm{~kJ} \mathrm{~mol}^{-1}$ for the dolomite. Using nitrogen porosimetry it was possible to observe that the dolomite presents mesopores of $0.03 \mu \mathrm{m}$, while the calcite presents mesopores of $0.01 \mu \mathrm{m}$. The evaluation of limestones porous structure together with their kinetic parameters, allowed concluding that the diffusion mechanism follows Fick law and Knudsen law for dolomite and calcite, respectively.
\end{abstract}

Keywords: diffusion mechanism; limestone; dessulfurization.

\section{INTRODUÇÃO}

O crescimento contínuo do consumo de energia está diretamente relacionado com a estrutura produtiva do país e o adequado suprimento de energia pode também levar a um avanço tecnológico e melhoria da qualidade de vida.

No Brasil, a partir da década de 90, o crescimento da oferta de energia se tornou insuficiente para fazer frente ao crescimento da demanda, especialmente em algumas importantes regiões do país. Embora a matriz energética brasileira seja majoritariamente de hidroelétrica, estes recursos se aproximam da exaustão ou se mostram ambientalmente inviáveis em algumas regiões. ${ }^{1}$

Dentro deste contexto, o carvão mineral, utilizado com as devidas precauções e tecnologias adequadas, pode ser uma alternativa para o problema energético brasileiro, pois segundo o Ministério da Ciência e Tecnologia (MCT) o Brasil possui reservas de aproximadamente 31 bilhões de toneladas de carvão mineral. Porém, os carvões brasileiros possuem teores de cinza que variam de 40 a $60 \%$ e altos índices de enxofre (de 1 a $6 \%)^{2}$

A queima de combustíveis fósseis para a produção de energia é considerada uma das principais fontes de emissão de $\mathrm{SO}_{2}$ e o acúmulo deste gás na atmosfera aumenta a acidez da água da chuva, acarretando no rompimento do equilíbrio ambiental. Contudo, há uma crescente preocupação em relação à preservação do planeta e a poluição atmosférica é ponto crucial neste contexto.

A combustão de carvões que possuem altos teores de enxofre e cinza é problemática em plantas convencionais. Assim, a possibilidade de se utilizarem reatores de leito fluidizado se torna promissora e este é um assunto que tem sido estudado por vários autores e com mais intensidade a partir da década de $1970 .{ }^{3}$ Neste processo, considerado tecnologia limpa do carvão, é possível se obterem significativas reduções de emissões poluentes, pois permite a adição de calcários, que além de serem eficientes sorventes de $\mathrm{SO}_{2}$, apresentam um baixo custo.

Outra possibilidade é a adição de biomassa em processos de combustão, porém sua adição isoladamente pode não ter as propriedades combustíveis adequadas devido a vários fatores como, por exemplo, o baixo poder calorífico, em função de sua própria natureza. Entretanto, pode ser empregada em misturas com carvão mineral, denominadas

*e-mail: danielamortari@yahoo.com.br blends, que nas proporções adequadas poderão apresentar bons resultados energéticos. ${ }^{4}$

A combustão de carvões ou blends de carvão/biomassa em reatores de leito fluidizado pode ocorrer entre 800 e $900{ }^{\circ} \mathrm{C} .{ }^{5} \mathrm{Nesta}$ faixa de temperatura se evita a formação de óxidos de nitrogênio $\left(\mathrm{NO}_{\mathrm{x}}\right)$ e também permite que ocorra a calcinação completa de calcários, formando óxidos de cálcio $(\mathrm{CaO})$ e de magnésio $(\mathrm{MgO})$ (Equação 1).

$$
\mathrm{CaMg}\left(\mathrm{CO}_{3}\right)_{2}(\mathrm{~s}) \rightarrow \mathrm{CaO}(\mathrm{s})+\mathrm{MgO}(\mathrm{s})+2 \mathrm{CO}_{2}(\mathrm{~g})
$$

Neste processo há a liberação de dióxido de carbono $\left(\mathrm{CO}_{2}\right)$ fazendo com que a estrutura física do calcário calcinado se torne mais porosa, aumentando a área superficial e, consequentemente, maior quantidade de reagente sólido disponível.

A combustão do carvão mineral promove a geração do gás $\mathrm{SO}_{2}$ e na presença do calcário calcinado ocorre a reação heterogênea gás-sólido, produzindo os sulfatos de cálcio $\left(\mathrm{CaSO}_{4}\right)$ e de magnésio $\left(\mathrm{MgSO}_{4}\right)$. Esta reação é denominada sulfatação e está apresentada na Equação 2.

$\mathrm{CaO}(\mathrm{s})+\mathrm{MgO}(\mathrm{s})+2 \mathrm{SO}_{2}(\mathrm{~g})+\mathrm{O}_{2}(\mathrm{~g}) \rightarrow \mathrm{CaSO}_{4}(\mathrm{~s})+\mathrm{MgSO}_{4}(\mathrm{~s})$

A reação de sulfatação resulta em um produto sólido $\left(\mathrm{CaSO}_{4} /\right.$ $\mathrm{MgSO}_{4}$ ) com volume cerca de três vezes maior que os respectivos reagente $\mathrm{CaO}$ e $\mathrm{MgO}$ e este processo leva a um bloqueio parcial ou total do interior das partículas, impedindo a completa conversão. ${ }^{6}$

A compreensão do mecanismo e do fator limitante das taxas de reação entre o $\mathrm{CaO}$ e $\mathrm{SO}_{2}$ tem sido objeto de muitas pesquisas. No início da década de 70 foram feitos os primeiros trabalhos demonstrando a influência da estrutura interna dos poros das partículas de $\mathrm{CaO}$ na reação com $\mathrm{SO}_{2}$. Com isso, passou-se a considerar aspectos físicos intrínsecos, e não apenas efeitos superficiais externos como até então considerados. ${ }^{7}$ Vários trabalhos são reportados na literatura relacionados a estudos de sorção de $\mathrm{SO}_{2}$ por calcários em sistemas termogravimétricos.

Dam-Johansen e Ostergaard ${ }^{8}$ estudaram a relação entre a compactação dos calcários e o seu tempo de calcinação e notaram que os calcários mais porosos são mais reativos, devido à facilidade de liberação do $\mathrm{CO}_{2}$, indicando que o transporte intrapartícula de $\mathrm{CO}_{2}$ é o determinante da taxa no processo de calcinação. 
Adánez et al. ${ }^{9}$ estudaram o efeito da porosidade de sorventes na sua capacidade de sulfatação. Concluíram que sorventes com uma distribuição de poros maiores que $100 \AA$ mostraram maior reatividade e capacidade de sulfatação. Sorventes com poros pequenos (menores que $80 \AA$ ) mantiveram-se inalterados ao longo do processo, devido ao bloqueio dos poros durante a sulfatação.

Além da porosidade e estrutura física dos sorventes, parâmetros cinéticos também são relevantes em estudos envolvendo difusão. Iisa e Hupa ${ }^{10}$ apresentaram um estudo cinético da reação de sulfatação empregando termogravimetria. Concluíram que para as partículas maiores, a sulfatação é limitada pela difusão através das camadas de produto, enquanto para as partículas menores, a sulfatação é controlada pela cinética da reação química.

Estudos previamente realizados pelo Núcleo de Engenharia Térmica e Fluidos - NETeF (EESC-USP), apresentam resultados de energia de ativação relacionando com o tipo de difusão do gás reagente $\left(\mathrm{SO}_{2}\right)$ que ocorre nos poros das partículas dos calcários calcinados..$^{11,12}$

Em complemento aos estudos anteriores do NETeF, o presente trabalho tem como objetivo apresentar uma interpretação do mecanismo de difusão do processo heterogêneo gás-sólido envolvido na reação de dessulfurização.

\section{Fundamentos de transferência de massa}

A sulfatação é um processo que envolve uma reação heterogênea em que o reagente gasoso se difunde da superfície externa para o interior da partícula de calcário e a reação ocorre na superfície de contato. A força motriz da difusão é originada pelo gradiente de concentração, que faz com que um componente da mistura de uma região de alta concentração se difunda para uma região de baixa concentração.

O fluxo molar total $W_{A}$ de uma espécie $\left(\mathrm{mol} / \mu^{2} \mathrm{~m}^{2} \mathrm{~s}\right)$, no caso o gás $\mathrm{SO}_{2}$ é denominado nesta descrição como espécie $A$, é resultado de duas contribuições: do fluxo de difusão molecular $\left(J_{A}\right)$, relativo ao movimento produzido por um gradiente de concentração $\left(\mathrm{mol} / \mu \mathrm{m}^{2} \mathrm{~s}\right)$, e do fluxo resultante do movimento da mistura gasosa $B_{A}\left(\mathrm{~mol} / \mu \mathrm{m}^{2} \mathrm{~s}\right)$ (Equação 3). ${ }^{13}$

$$
W_{A}=J_{A}+B_{A}
$$

O fluxo molar total do gás também pode ser expresso em termos da concentração $C_{A}\left(\mathrm{~mol} / \mu \mathrm{m}^{3}\right)$ (Equação 4$)$.

$$
W_{A}=J_{A}+C_{A} V
$$

sendo $V$ a velocidade molar média $(\mu \mathrm{m} / \mathrm{s})$.

O fluxo molar total também pode ser expresso em termos de fração molar de $A$ (Equação 5).

$$
W_{A}=J_{A}+y_{A}\left(W_{A}+W_{B}\right)
$$

sendo $y_{A}$ a fração molar da espécie $A$.

$W_{A}$ pode ser dado pelo produto $\left(C_{A} V_{A}\right)$, sendo $\left(V_{A}\right)$ a velocidade da espécie química $A$. $W_{B}$ representa o $\mathrm{O}_{2}$ envolvido na reação de dessulfurização, como descrito na Equação 2.

A velocidade da espécie química $A$ é calculada pela Equação da teoria cinética (Equação 6).

$$
V_{A}=\sqrt{\frac{8 R T}{\pi M}}
$$

A velocidade média molar $V$ é dada pela Equação 7 .

$$
V=y_{A} V_{A}+y_{B} V_{B}
$$

sendo $y_{B}$ a fração molar da espécie $B$ e $V_{B}$ a velocidade de partícula da espécie $B$.

A equação constitutiva para $J_{A}$, que é o fluxo difusional resultante de uma diferença de concentração, está relacionada ao gradiente de concentração pela primeira lei de Fick (Equação 8):

$$
J_{A}=-c D_{A B} \nabla y_{A}
$$

sendo $c$ a concentração total $\left(\mathrm{mol} / \mu \mathrm{m}^{3}\right), D_{A B}$ a difusividade de $A$ em $B\left(\mu \mathrm{m}^{2} / \mathrm{s}\right)$.

\section{PARTE EXPERIMENTAL}

\section{Estudo cinético empregando termogravimetria}

Foram estudados dois tipos de calcário nacionais: calcítico e dolomítico - denominados por CI e DP, respectivamente. A Tabela 1 apresenta a composição química para ambos os calcários.

Tabela 1. Composição química (\% massa/massa) dos calcários calcítico (CI) e dolomítico (DP)

\begin{tabular}{lccccccc}
\hline Calcário & $\mathrm{Ca}$ & $\mathrm{Mg}$ & $\mathrm{Fe}$ & $\mathrm{Al}$ & $\mathrm{Sr}$ & $\mathrm{K}$ & $\mathrm{Mn}$ \\
\hline CI & 35,34 & 0,35 & 2,45 & 0,25 & 0,14 & 0,09 & 0,52 \\
$\mathrm{DP}$ & 17,07 & 11,73 & 0,32 & 0,42 & 0,08 & 0,13 & 0,09 \\
\hline
\end{tabular}

A granulometria média de $545 \mu \mathrm{m}$ dos calcários foi obtida por meio de seleção entre duas peneiras sucessivas da série ASTM, de modo a compor a faixa mais estreita possível e minimizar o efeito da distribuição granulométrica.

Os valores de energia de ativação $\left(E_{a}\right)$ foram obtidos em trabalho anterior, ${ }^{11}$ por meio de ensaios termogravimétricos (TG). Nestes testes as amostras do calcário natural foram inseridas em suporte de alumina e introduzidas no forno da balança termogravimétrica. Inicialmente foi feita a calcinação da amostra em atmosfera dinâmica de ar sintético $\left(80 \mathrm{~mL} \mathrm{~min}^{-1}\right)$, com razão de aquecimento de $30{ }^{\circ} \mathrm{C}$ $\min ^{-1}$ até a temperatura desejada, ou seja, a temperatura na qual se realiza o teste de sulfatação. Para isto foram selecionadas quatro temperaturas diferentes: $750,800,850$ e $900{ }^{\circ} \mathrm{C}$.

Após o aquecimento e atingida a temperatura do teste, a amostra encontrou-se calcinada. Nesta temperatura aplicou-se uma condição isotérmica de 30 min. Após os 5 min de estabilização, o gás $\mathrm{SO}_{2}$ foi injetado no forno da balança com vazão de $20 \mathrm{~mL} \mathrm{~min}^{-1}$, conferindo uma fração de $\mathrm{SO}_{2}$ de $20 \%$ da atmosfera total. O emprego de alta concentração de $\mathrm{SO}_{2}$, condição denominada diferencial, é aplicada de modo a considerar os efeitos intrínsecos do processo, eliminando as limitações difusivas.

A partir destes testes, calculou-se a energia de ativação para os calcários utilizando-se a Equação de Arrhenius (Equação 9).

$$
k(T)=A \exp \left(-\frac{E_{a}}{R T}\right)
$$

sendo $A$ o fator pré-exponencial ou fator de frequência, $E_{a}$ a energia de ativação aparente $\left(\mathrm{kJ} \mathrm{mol}^{-1}\right), R$ a constante universal dos gases $\left(\mathrm{J} \mathrm{K}^{-1} \mathrm{~mol}^{-1}\right)$ e $T$ a temperatura $(\mathrm{K})$.

Empregando-se as curvas derivadas (DTG) obtidas para todas as condições estudadas na balança termogravimétrica, se obtém o valor de dm/dt (pico da curva DTG), ponto onde a taxa de reação é máxima.

Foi determinado o coeficiente de taxa de reação máxima $\left(k_{\max }\right)$, de acordo com a Equação 10.

$$
k_{\max }=\frac{1}{m} \frac{d m}{d t}
$$


sendo $m$ a massa transiente correspondente às frações de Ca e $\mathrm{Mg}$ disponíveis no calcário para a reação, sendo obtida por meio da Equação 11.

$$
m=M_{A}\left(Y_{C a}+Y_{M g}\right)-\left\{\left(M-M_{c}\right)\left[\frac{\left(W_{C a} Y_{C a}+W_{M g} Y_{M g}\right)}{\left(W_{S O_{2}}+1 / 2 W_{O_{2}}\right)\left(Y_{C a}+Y_{M g}\right)}\right]\right\}
$$

sendo $M_{A}$ a massa inicial da amostra (calcário natural), $M$ a massa transiente da amostra durante a sulfatação, $M_{C}$ a massa do calcário calcinado, $Y$ a fração em massa de $\mathrm{Ca}$ e $\mathrm{Mg}$ no calcário natural, $W$ a massa molar de cada um dos componentes $\left(\mathrm{Ca}, \mathrm{Mg}, \mathrm{SO}_{2}\right.$ e $\left.\mathrm{O}_{2}\right)$.

Das Equações 9 e 10 obtém-se:

$$
\ln \left[-\left(\frac{1}{m} \frac{d m}{d t}\right)_{\max }\right]=\ln A-\frac{E_{a}}{R} \frac{1}{T}
$$

A representação gráfica do conjunto de pontos obtidos para $\ln \left[-\left(\frac{1}{m} \frac{d m}{d t}\right)_{\max }\right]$ em função do inverso da temperatura fornece os parâmetros cinéticos de Arrhenius pelo ajuste da reta. Os valores do fator pré-exponencial $A\left(\mathrm{~s}^{-1}\right)$ e da energia de ativação aparente $E_{a}$ foram obtidos, respectivamente, pelo coeficiente linear e coeficiente angular. Os resultados obtidos, bem como a metodologia aplicada, foram apresentados em detalhes no trabalho de Ávila et al.. ${ }^{11}$

\section{Determinação de área superficial específica e distribuição de diâmetro de poros}

Dados das características físicas dos calcários foram determinados utilizando-se um porosímetro por adsorção de nitrogênio $\left(\mathrm{N}_{2}\right)$ da Micromeritics (ASAP 2020). Em todos os ensaios foram obtidos dados de: área superficial específica calculada pela equação de BET (Brunauer, Emmett e Teller), diâmetro médio de poros na etapa de adsorção e dessorção e volume médio de poros na etapa de adsorção e dessorção. O volume total de poros e o diâmetro médio de poros fornecidos pelo software do equipamento foram calculados utilizando-se o algoritmo do método BJH (Barrett, Joyner e Halenda).

\section{RESULTADOS}

Quando o calcário $\left(\mathrm{CaMg}\left(\mathrm{CO}_{3}\right)_{2}\right)$ é submetido a um aquecimento, é possível verificar uma perda de massa devido à calcinação, onde ocorre a liberação de $\mathrm{CO}_{2}$ e a formação de óxido de cálcio $(\mathrm{CaO})$ e óxido de magnésio (MgO) (Equação 1). Esse processo se inicia a uma temperatura de aproximadamente $650{ }^{\circ} \mathrm{C}$ sob as condições de $30{ }^{\circ} \mathrm{C}$ min $^{-1}$ em balança termogravimétrica e se completa a aproximadamente $850^{\circ} \mathrm{C}$. Com a adição de $\mathrm{SO}_{2}$ para a etapa da sulfatação, verifica-se um ganho de massa devido à reação entre os óxidos e o gás $\mathrm{SO}_{2}$ injetado no forno da balança TG (Equação 2). Os perfis das curvas TG e DTG também podem ser observados como previamente descritos em Ávila et al..${ }^{11}$ Com base nesses valores de ganho de massa é possível avaliar a capacidade do calcário no processo de sorção do gás reagente.

A fim de avaliar a capacidade de sorção dos calcários, Ávila et al. ${ }^{11}$ calcularam a conversão no processo de sulfatação a $850{ }^{\circ} \mathrm{C}$, temperatura típica do processo de combustão em leito fluidizado. Este resultado pode ser evidenciado na Figura 1, que mostra a conversão percentual dos dois calcários estudados. O calcário DP foi aproximadamente $14 \%$ mais reativo quando comparado ao CI.

Com base nos resultados encontrados, supõe-se que a estrutura física do calcário DP é diferente da do CI. Esta suposição pode ser comprovada através das imagens obtidas por microscopia eletrônica de varredura (MEV), com ampliação de 5000 vezes, apresentadas nas Figuras 2 e 3 . Observa-se que as estruturas morfológicas dos calcários

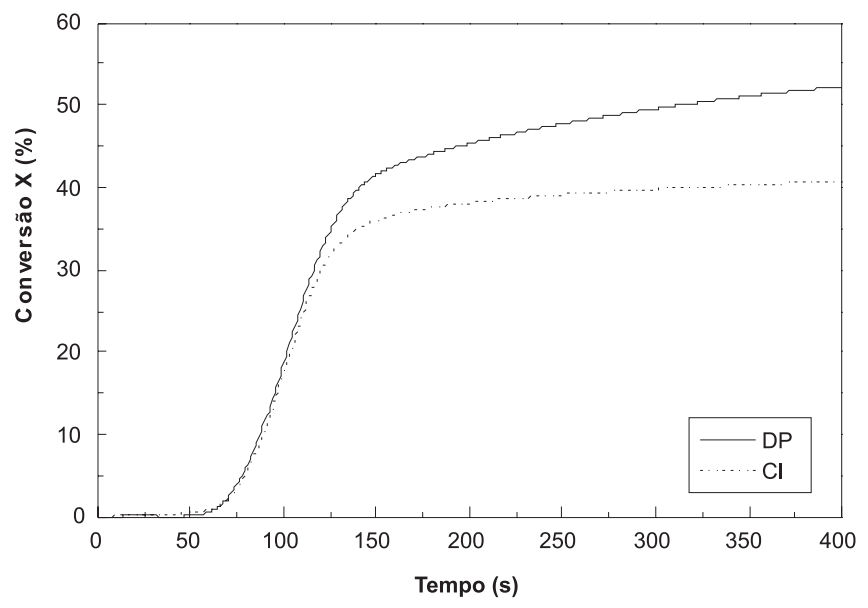

Figura 1. Conversão (X\%) em função do tempo (s) do processo de sulfatação

apresentam-se diferentes quanto à compactação e porosidade.

A Figura 2 mostra as imagens dos calcários CI (a) e DP (b) na forma natural. Nota-se que o calcário DP apresenta uma estrutura mais porosa em relação ao calcário CI. Após a calcinação (Figura 3) observa-se que a estrutura morfológica do calcário DP (b) se manteve após a calcinação. Já no calcário CI (a), que quando natural apresenta uma estrutura mais compacta, após a calcinação nota-se uma estrutura superficial mais porosa, semelhante ao calcário DP.

a)

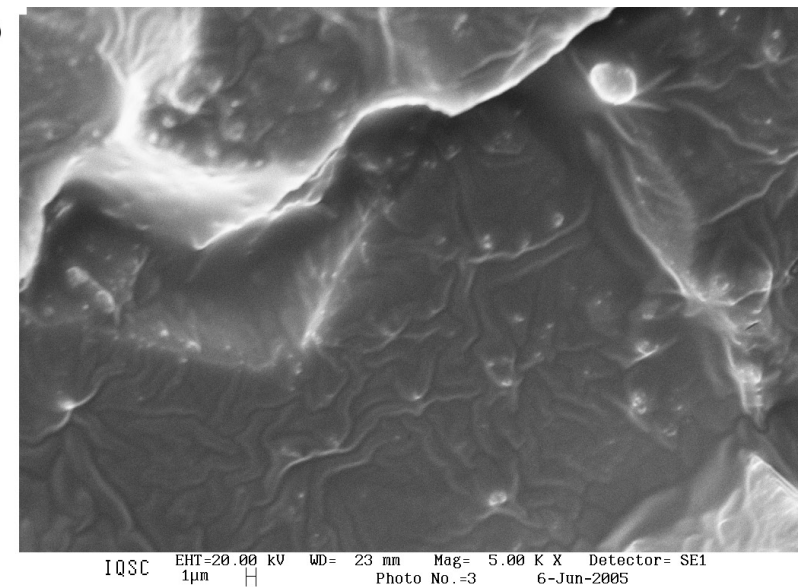

b)

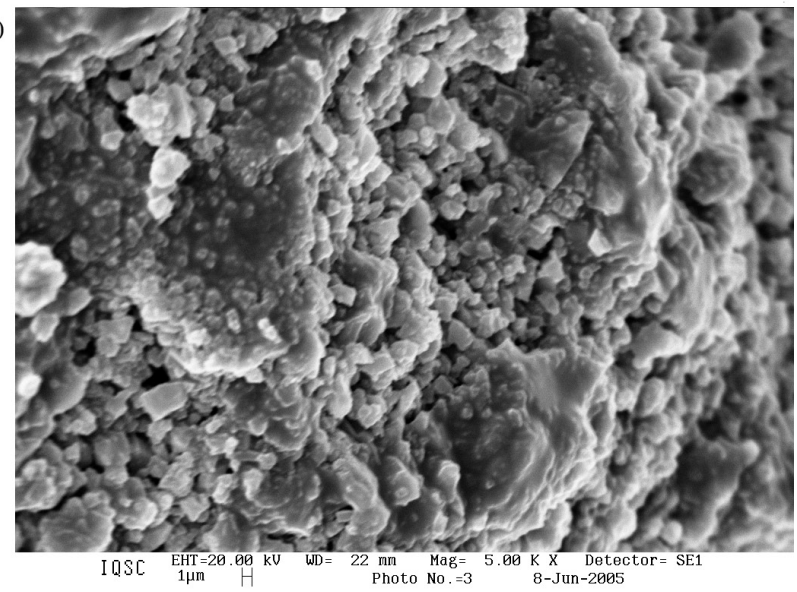

Figura 2. Estrutura porosa dos calcários calcítico (a) e dolomítico (b) na forma natural 

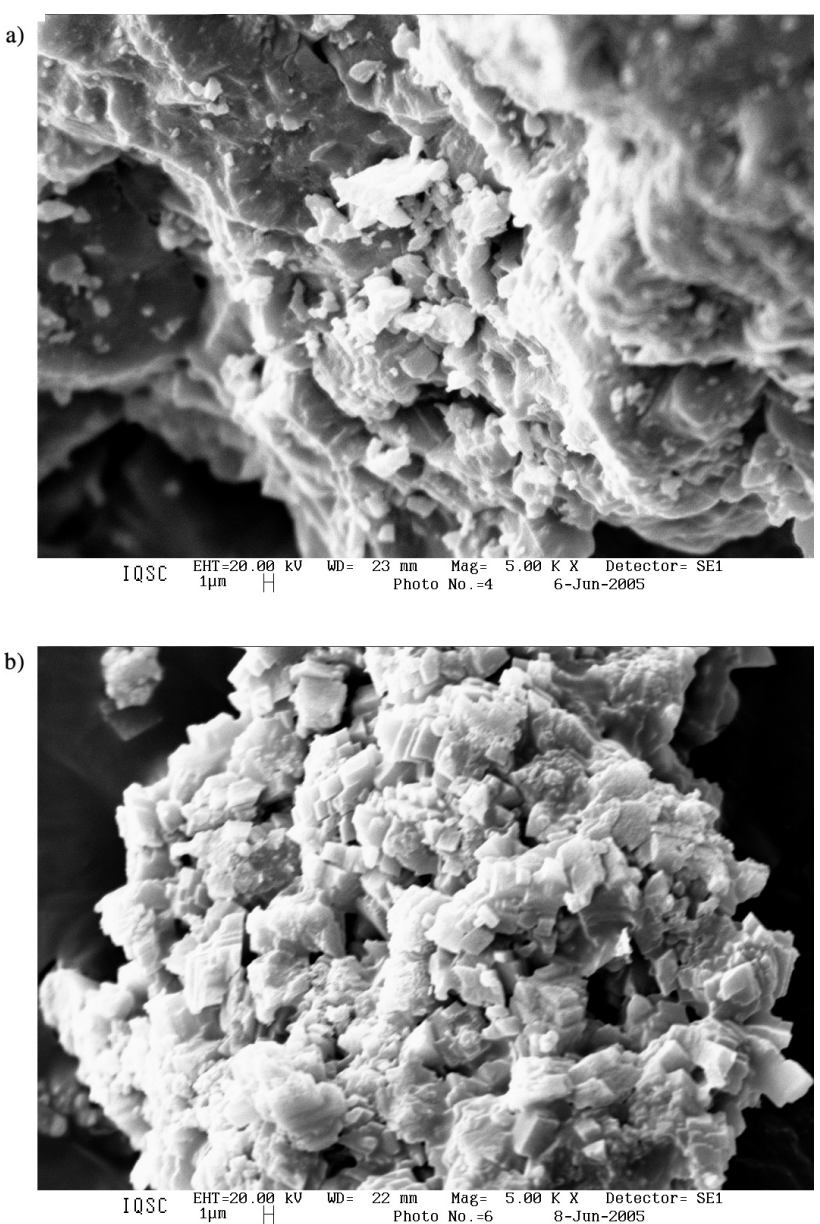

Figura 3. Estrutura porosa dos calcários calcítico (a) e dolomítico (b) na forma calcinada

Embora estas imagens permitam visualizar a diferença de porosidade, elas são apenas qualitativas. Para uma determinação quantitativa, utilizou-se a técnica de porosimetria por adsorção de $\mathrm{N}_{2}$.

As Figuras 4 e 5 apresentam as curvas (isotermas) de adsorção e dessorção de $\mathrm{N}_{2}$ para ambos os calcários, na forma natural e calcinada. Dados de porosidade, como área superficial BET, diâmetro médio de poros nas etapas de adsorção/dessorção $\left(\mathrm{D}_{\text {P.Ads }} / \mathrm{D}_{\text {P.Ds }}\right)$ e volume médio de poros nas etapas de adsorção/dessorção $\left(\mathrm{V}_{\text {P.Ads }} / \mathrm{V}_{\text {P.Ds }}\right)$ são apresentados na Tabela 2 .

Tabela 2. Parâmetros físicos para os calcários calcítico (CI) e dolomítico (DP) natural e calcinado

\begin{tabular}{lcccc}
\hline \multirow{2}{*}{ Parâmetros físicos } & \multicolumn{2}{c}{ Calcário natural } & \multicolumn{2}{c}{ Calcário calcinado } \\
& $\mathrm{CI}$ & $\mathrm{DP}$ & $\mathrm{CI}$ & $\mathrm{DP}$ \\
\hline $\mathrm{S}_{\mathrm{BET}} / \mathrm{m}^{2} \mathrm{~g}^{-1}$ & 2,86 & 2,78 & 13,28 & 8,98 \\
$\mathrm{D}_{\mathrm{PAD}} / \mu \mathrm{m}$ & 0,0185 & 0,0179 & 0,0119 & 0,0309 \\
$\mathrm{D}_{\mathrm{PD}} / \mu \mathrm{m}$ & 0,0121 & 0,0097 & 0,0107 & 0,0265 \\
$\mathrm{~V}_{\mathrm{P}} / \mathrm{cm}^{3} \mathrm{~g}^{-1}$ & 0,010493 & 0,007204 & 0,032251 & 0,0568 \\
\hline
\end{tabular}

Nota-se que após a calcinação houve um aumento significativo da área superficial BET dos calcários, de 364\% para o CI e de $223 \%$ para o DP. Como esperado, estes resultados indicam que a calcinação promove um aumento significativo da área disponível para a reação, porém um parâmetro que se deve levar em consideração é principalmente o desenvolvimento dos poros.

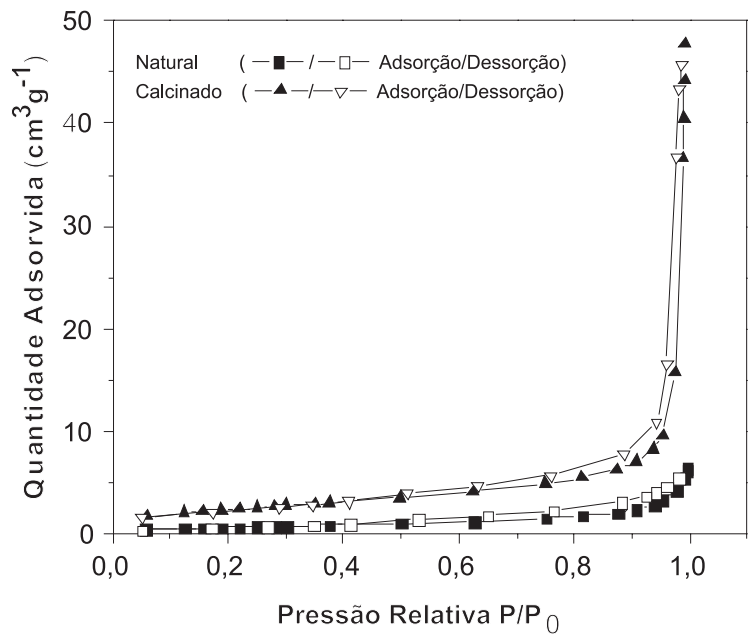

Figura 4. Isotermas de adsorção para o calcário dolomítico (DP) na forma natural e calcinada

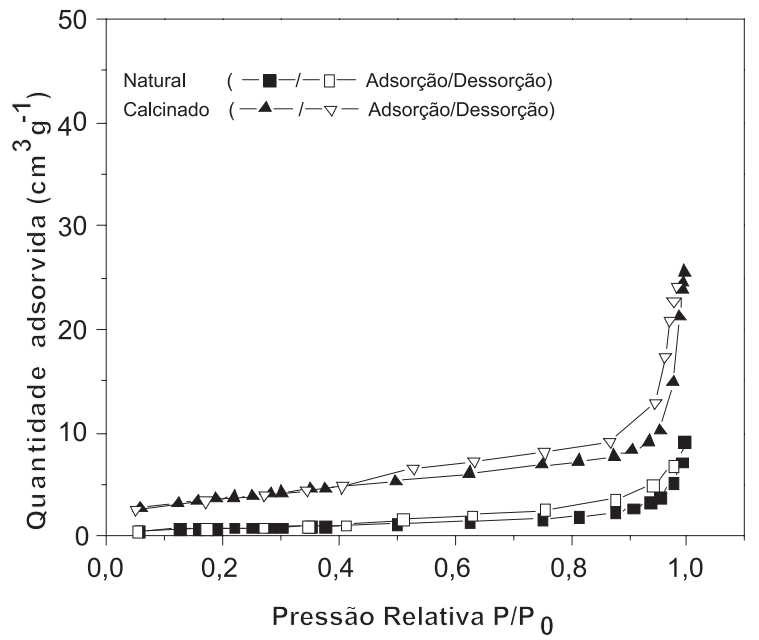

Figura 5. Isotermas de adsorção para o calcário calcítico (CI) na forma natural e calcinada

Comparando-se os dados apresentados na Tabela 2, nota-se que após a calcinação, enquanto no calcário CI há uma diminuição do diâmetro dos poros $\left(\mathrm{D}_{\mathrm{P} . \mathrm{AD}} / \mathrm{D}_{\mathrm{P.D}}\right)$, para o calcário $\mathrm{DP}$ há um aumento do diâmetro dos poros. Levando-se em consideração que o calcário DP é mais reativo que o CI, como mostrado na Figura 1, há uma indicação de que a estrutura morfológica desempenha um papel importante na intensidade da reação de sorção.

Quanto ao processo reativo da sorção de $\mathrm{SO}_{2}$, os parâmetros de Arrhenius obtidos mostraram que a energia de ativação determinada para o calcário CI foi aproximadamente $4 \mathrm{~kJ} \mathrm{~mol}^{-1}$. ${ }^{11}$ De acordo com dados encontrados na literatura, este valor indica que a sulfatação do calcário CI é controlada por difusão de Knudsen. Em contrapartida, o valor de energia de ativação da ordem obtida para o calcário DP, de aproximadamente $12 \mathrm{~kJ} \mathrm{~mol}^{-1}$, corresponde a um mecanismo satisfatoriamente explicado considerando a difusão molecular. ${ }^{14} \mathrm{O}$ valor da $E_{a}$ para o calcário DP foi 3 vezes maior que o obtido para o calcário CI, ou seja, o calcário DP necessita de aproximadamente 3 vezes mais energia para que a reação ocorra.

A diferença observada entre a conversão no processo de sulfatação e os valores encontrados para energia de ativação sugere que a estrutura física é um fator importante na reatividade do sorvente calcário no processo de sulfatação, afetando diretamente a cinética da reação gás-sólido. De acordo com os resultados referentes à es- 
trutura porosa, pode-se dizer que o tamanho dos poros é um aspecto importante nesta variação de reatividade.

Na reação de dessulfurização, o reagente $\mathrm{SO}_{2}$ se difunde nos poros do calcário, sendo que a concentração deste na entrada do poro é maior do que no seu interior. Como o mecanismo de difusão depende da estrutura porosa do sólido, em cada calcário com estrutura diferente a difusão seguirá uma determinada lei de mecanismo. No caso de calcários meso e macroporosos (>0,002 $\mu \mathrm{m})$, a difusão segue a lei de Fick - também conhecida como difusão molecular, descrita na Equação 8. Neste caso, as moléculas do gás colidem mais entre si do que com a parede do sólido, justificando um maior valor da energia de ativação $\left(12 \mathrm{~kJ} \mathrm{~mol}^{-1}\right)$ quando comparado à energia de ativação determinada para o calcário $\mathrm{CI}\left(4 \mathrm{~kJ} \mathrm{~mol}^{-1}\right)$. No entanto, há uma indicação de que a mesoporosidade do calcário DP permite maiores conversões antes que ocorra o bloqueio dos poros.

No caso de calcários que apresentam majoritariamente microporosos em sua estrutura $(<0,002 \mu \mathrm{m})$, a velocidade da mistura gasosa é muito pequena, assim o termo $B_{A}$ da Equação 3 é desprezível quando comparado a $J_{A}$, e a introdução do gás para o interior das partículas é mais significativa pelo processo da difusão e controlada cineticamente. Desta forma a Equação 3 é reduzida à Equação 13.

$$
W_{A}=J_{A}
$$

A difusão nestas condições, chamada de difusão de Knudsen, ocorre quando o livre caminho médio entre moléculas do gás é da mesma ordem do diâmetro de poros do sólido. ${ }^{13}$ Neste caso, as moléculas colidem mais frequentemente com as paredes dos poros do que entre si. Como a reação ocorre na superfície de contato, torna justificáveis os menores valores de energia de ativação para o calcário CI quando comparado ao calcário DP.

O calcário CI calcinado por apresentar mesoporos com diâmetro médio de $0,01 \mu \mathrm{m}$, durante o processo de dessulfurização o bloqueio dos poros pode ocorrer mais rapidamente, consequentemente, são obtidas conversões mais baixas no processo de sorção de $\mathrm{SO}_{2}$.

\section{CONCLUSÕES}

A reação gás-sólido $\left(\mathrm{SO}_{2}-\mathrm{CaO}\right)$ é conduzida tanto por aspectos químicos quanto físicos e a energia de ativação é um parâmetro que indica a viabilidade da reação. No entanto, a reação química é limitada pela difusão do $\mathrm{SO}_{2}$ e $\mathrm{O}_{2}$ para o interior das partículas de calcário.

$\mathrm{Na}$ difusão, o fator limitante é a estrutura física do calcário e um fator relevante neste processo é o tamanho dos poros. Os resul- tados mostraram que para o calcário dolomítico calcinado (DP), por apresentar mesoporos na região entre 0,02 e $0,03 \mu \mathrm{m}$, a difusão se comporta de acordo com a Lei de Fick, pois a energia de ativação foi da ordem de $12 \mathrm{~kJ} \mathrm{~mol}^{-1}$. Para estruturas com poros menores que $0,01 \mu \mathrm{m}$, apresentadas no calcário calcítico calcinado, a energia de ativação resultou em 4 kJ mol, portanto, seguindo a Lei de Knudsen.

\section{MATERIAL SUPLEMENTAR}

A curva termogravimétrica característica do processo calcinação seguida pela sulfatação do calcário está disponível, com acesso livre, na forma de arquivo PDF, em http://quimicanova.sbq.org.br.

\section{AGRADECIMENTOS}

À FAPESP (Processo 2008/07215 e 2008/54062-9) e a CAPES (Processo PNPD 34088) pelo apoio financeiro.

\section{REFERÊNCIAS}

1. Vieira, J. M.; Sodré, E.; Leal, N.; Guedes, D. F.; Alves, F.; Melo, G. H. S.; Proceeding of XIII ERIAC - Décimo Tercer Encuentro Regional Ibero Americano de Cigré, Puerto Iguazú, Argentina, 2009.

2. Süffert, T.; Carvão nos Estados do Rio Grande do Sul e Santa Catarina, CPRM: Porto Alegre, 1997.

3. Jacobs, J. P.; Chem. Eng. Sci. 1999, 54, 5559.

4. Biagini, E.; Lippi, F.; Petarca, L.; Tognotti, L.; Fuel 2002, 81, 1041.

5. Fuertes, A. B.; Marban, G.; Rubiera, F.; Trans. IChemE. 1993, 71, 421.

6. Zarkanitis, S.; Sotirchos, S. V.; AIChE J. 1989, 35, 821.

7. Borgwardt, R. H.; Bruce, K. R.; AIChE J. 1986, 31, 103.

8. Dam-Johansen, K. Ostergaard, K.; Chem. Eng. Sci. 1991, 46, 827.

9. Adánez, J.; Fierro, V.; De Diego, J.A.; García-Labiano, F.; Thermochim. Acta 1996, 277, 151.

10. Iisa, K.; Hupa, M.; J. Energy Inst. 1992, 65, 201.

11. Ávila, I. ; Crnkovic, P. M.; Milioli, F. E.; Quim. Nova 2007, 30, 1275.

12. Crnkovic, P. M.; Ávila, I.; Milioli, F. E.; Pagliuso, J. D.; Proceeding of International Mechanical Engineering Congress and Exposition, Boston, United States, 2008.

13. Fogler H. S.; Elementos da engenharia das reações químicas, $3^{\mathrm{a}}$ ed., LTC: Rio de Janeiro, 2002.

14. Fuertes, A. B.; Velasco, G.; Fernandez, M. J.; Alvarez, T.; Thermochim. Acta 1994, 242, 161. 


\section{INTERPRETAÇÃO DO MECANISMO DE DIFUSÃO NO PROCESSO GÁS-SÓLIDO DA REAÇÃO DE DESSULFURIZAÇÃO}

Daniela Andresa Mortari*, Paula Manoel Crnkovic, Ivonete Ávila e Fernando Eduardo Milioli

Departamento de Engenharia Mecânica, Escola de Engenharia de São Carlos, Universidade de São Paulo, CP 780. 13560-970 São Carlos - SP, Brasil

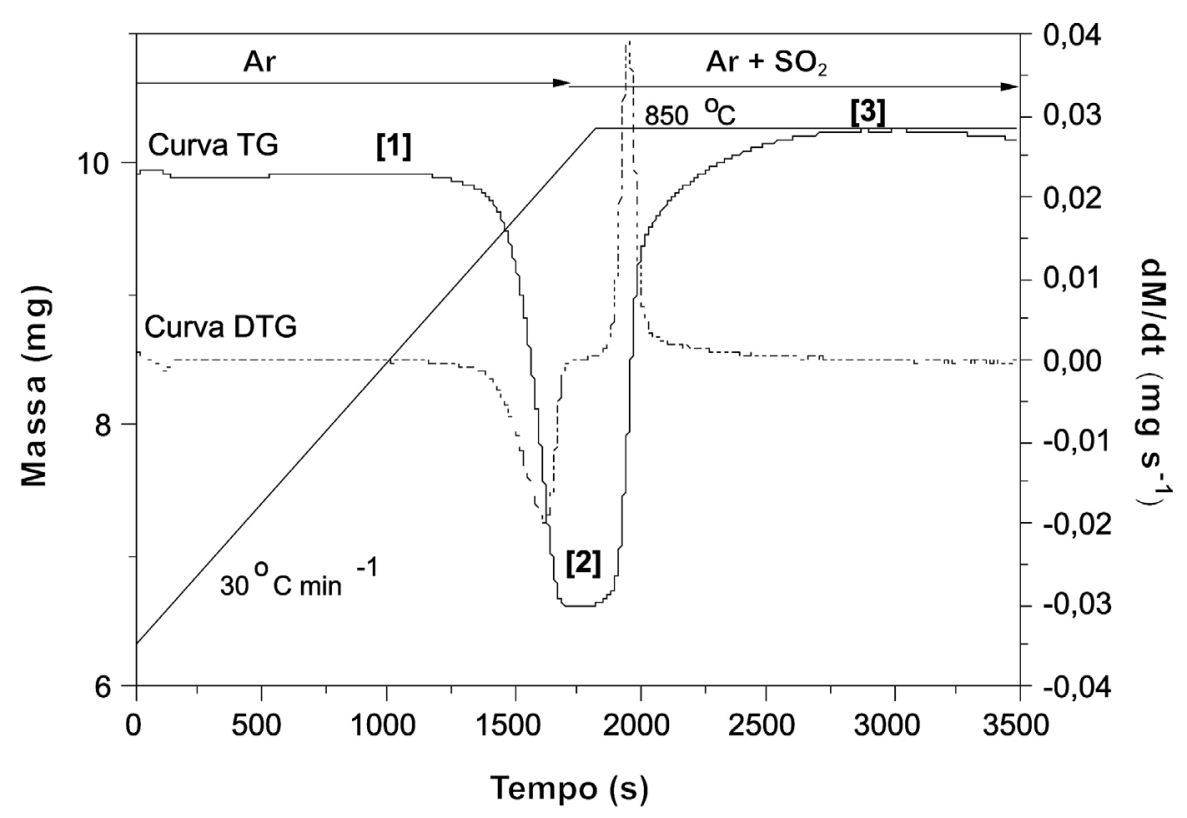

Figura 1S. Curva termogravimétrica da calcinação seguida de sulfatação do calcário DP a $850^{\circ} \mathrm{C}$ 